Homology, Homotopy and Applications, vol.4(1), 2002, pp.59-69

\title{
A HIGHER DIMENSIONAL HOMOTOPY SEQUENCE
}

\author{
M. GRANDIS AND E. M. VITALE \\ (communicated by Hvedri Inassaridze)
}

\begin{abstract}
We associate to a continuous map between pointed spaces a long 2-exact sequence of homotopy pointed groupoids. The usual homotopy sequence of a map follows from this 2-exact sequence taking, for each groupoid, the set of connected components. We also study a condition of strong 2-exactness for a sequence of cat-groups and pointed groupoids.
\end{abstract}

\section{Introduction}

Let us start with an old story. The simplest homotopy invariant of a pointed topological space $Y$ is the pointed set $\pi_{0}(Y)$ of its path-connected components. Using the loop functor $\Omega$, we have a family of pointed sets $\pi_{n}(Y)=\pi_{0}\left(\Omega^{n} Y\right)$ which are (abelian) groups for $n \geqslant 1(n \geqslant 2)$. If $f: X \rightarrow Y$ is a continuous map preserving the base point, these homotopy invariants fit into a long exact sequence of groups and pointed sets

$$
\begin{gathered}
\ldots \rightarrow \pi_{n}(K f) \rightarrow \pi_{n}(X) \rightarrow \pi_{n}(Y) \rightarrow \ldots \\
\ldots \rightarrow \pi_{1}(K f) \rightarrow \pi_{1}(X) \rightarrow \pi_{1}(Y) \rightarrow \pi_{0}(K f) \rightarrow \pi_{0}(X) \rightarrow \pi_{0}(Y)
\end{gathered}
$$

where $K f \rightarrow X$ is the homotopy kernel of $f$.

The aim of this short note is to show that this sequence is a kind of "projection" on the category of pointed sets of an exact sequence of higher dimensional homotopy invariants. In fact the first two invariants of $Y, \pi_{0}(Y)$ and $\pi_{1}(Y)$, can be interpreted, respectively, as the set of connected components and the group of automorphisms at the base point of the fundamental groupoid $\Pi_{1}(Y)$. Using once again the loop functor, one obtains a family of pointed groupoids $\Pi_{n+1}(Y)=\Pi_{1}\left(\Omega^{n} Y\right)$. We will show that these homotopy invariants fit into a sequence of pointed groupoids and pointed functors

$$
\begin{gathered}
\ldots \rightarrow \Pi_{n}(K f) \rightarrow \Pi_{n}(X) \rightarrow \Pi_{n}(Y) \rightarrow \ldots \\
\ldots \rightarrow \Pi_{2}(K f) \rightarrow \Pi_{2}(X) \rightarrow \Pi_{2}(Y) \rightarrow \Pi_{1}(K f) \rightarrow \Pi_{1}(X) \rightarrow \Pi_{1}(Y)
\end{gathered}
$$

which is "2-exact", i.e. exact in a suitable categorical sense (see Definition 1). Taking, for each pointed groupoid of this sequence, the pointed set of its connected components, one comes back to the classical exact sequence of the map $f$.

Received February 4, 2002, revised June 7, 2002; published on June 25, 2002.

2000 Mathematics Subject Classification: 18G55, 20L05, 55Q05.

Key words and phrases: Homotopical algebra, fundamental groupoids, homotopy sequences.

(C) 2002, M. Grandis and E. M. Vitale. Permission to copy for private use granted. 
The second part of this paper is devoted to study a condition of strong 2-exactness which can be stated when a cat-group acts on a pointed groupoid. It is well-known that the classical homotopy sequence has a strong exactness at the transition point between groups and pointed sets

$$
\pi_{1}(Y) \rightarrow \pi_{0}(K f) \rightarrow \pi_{0}(X)
$$

which is stated in terms of the action of the group $\pi_{1}(Y)$ on the pointed set $\pi_{0}(K f)$. We show that the higher dimensional homotopy sequence satisfies a similar condition. We state our condition using a suitable 2-dimensional colimit, which is nothing but the cokernel (in the sense of bilimits) when the sequence is a sequence of symmetric cat-groups. This provide also a new interpretation of the strong exactness for a sequence of groups and pointed sets.

A warning: the composite of two arrows $f: X \rightarrow Y$ and $g: Y \rightarrow Z$ in a category is denoted by $f \cdot g$.

\section{Preliminaries}

In this section we recall all the ingredients we need for the sequence of groupoids: the kernel of a morphism of pointed groupoids, the definition of 2-exactness, the homotopy equivariance of the fundamental groupoid of a space.

A pointed groupoid $\mathbb{G}=(\mathbb{G}, 0)$ is a groupoid $\mathbb{G}$ (that is a category in which each arrow is an isomorphism) together with a chosen object 0 . A morphism of pointed groupoids $F: \mathbb{G} \rightarrow \mathbb{H}$ is a functor with a specified arrow $f_{0}: 0 \rightarrow F(0)$ in $\mathbb{H}$ (a morphism $F: \mathbb{G} \rightarrow \mathbb{H}$ is strict if the arrow $f_{0}$ is the identity); a pointed natural transformation $\varphi: F \Rightarrow G: \mathbb{G} \rightarrow \mathbb{H}$ between morphisms of pointed groupoids is a natural transformation (necessarily a natural isomorphism) such that $f_{0} \cdot \varphi_{0}=g_{0}$. In this way we obtain a 2-category $G p d_{*}$.

Given a morphism $F: \mathbb{G} \rightarrow \mathbb{H}$ in $G p d_{*}$, its (homotopy) kernel $k F: K F \rightarrow \mathbb{G}$ can be described in the following way :

- an object of $K F$ is a pair $(X, x)$ with $X$ an object of $\mathbb{G}$ and $x: 0 \rightarrow F(X)$ an arrow in $\mathbb{H}$;

- an arrow $f:(X, x) \rightarrow\left(X^{\prime}, x^{\prime}\right)$ of $K F$ is an arrow $f: X \rightarrow X^{\prime}$ in $\mathbb{G}$ such that $x \cdot F(f)=x^{\prime}$;

- the base object of $K F$ is $\left(0, f_{0}\right)$;

- the functor $k F$ sends $f:(X, x) \rightarrow\left(X^{\prime}, x^{\prime}\right)$ to $f: X \rightarrow X^{\prime}$; it is a strict morphism.

There is a pointed natural transformation $\kappa F: 0 \Rightarrow k F \cdot F$ (where 0 is the constant morphism which sends each arrow to the identity of the base object of $\mathbb{H}$ ) given, at the point $(X, x)$, by $x: 0 \rightarrow F(X)$.
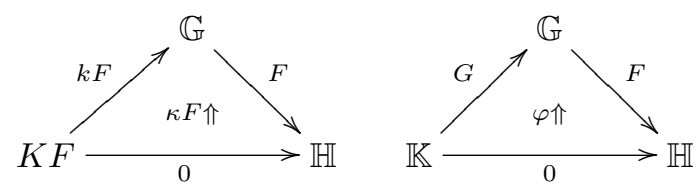
The triple $(K F, k F, \kappa F)$ has the following universal property : given any other triple $(\mathbb{K}, G, \varphi)$ in $G p d_{*}$ as in the previous diagram, there is a unique morphism $G^{\prime}: \mathbb{K} \rightarrow K F$ such that $G^{\prime} \cdot k F=G$ and $G^{\prime} \cdot \kappa F=\varphi$. The functor $G^{\prime}$ is defined by $G^{\prime}\left(g: Y \rightarrow Y^{\prime}\right)=G(g):\left(G(Y), \varphi_{Y}\right) \rightarrow\left(G\left(Y^{\prime}\right), \varphi_{Y^{\prime}}\right)$. The kernel $(K F, k F, \kappa F)$ has also a "biuniversal" property, studied in $[\mathbf{1 1}, \mathbf{1 6}]$, which characterizes it up to equivalence.

A suitable notion of exactness in $G p d_{*}$, introduced in $[\mathbf{1 1}, \mathbf{1 6}]$ to study some examples coming from ring theory, is given in the following definition, related to a notion of "homotopical exactness" studied in $[\mathbf{9}]$.

Definition 1. A triple $(G, \varphi, F)$ in $G p d_{*}$

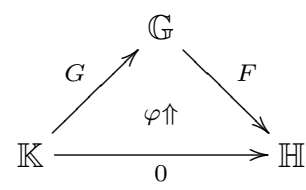

is 2-exact if the comparison morphism $G^{\prime}: \mathbb{K} \rightarrow K F$ is full and essentially surjective on objects.

If $\mathbb{G}=(\mathbb{G}, 0)$ is a pointed groupoid, we write $\pi_{0}(\mathbb{G})$ for the pointed set of isomorphism classes of objects, and $\pi_{1}(\mathbb{G})$ for the group of automorphisms $\mathbb{G}(0,0)$. If $\mathbb{G}$ is a (braided) categorical group, $\pi_{0} \mathbb{G}$ ) is a (abelian) group and $\pi_{1}(\mathbb{G})$ is abelian (see $[\mathbf{5}, \mathbf{1 0}]$ for the notion of (braided) categorical group). Both $\pi_{0}$ and $\pi_{1}$ give rise to functors on the underlying category of $G p d_{*}$

$$
\pi_{0}: G p d_{*} \rightarrow \operatorname{Set}_{*} \quad \pi_{1}: G p d_{*} \rightarrow \text { Groups }
$$

which are homotopy invariants, in the sense that if there is a 2-cell $\varphi: F \Rightarrow G: \mathbb{G} \rightarrow$ $\mathbb{H}$ in $G p d_{*}$, then $\pi_{0}(F)=\pi_{0}(G)$ and $\pi_{1}(F)=\pi_{1}(G)$. In particular, if $F$ is an equivalence in $G p d_{*}$, then $\pi_{0}(F)$ and $\pi_{1}(F)$ are isomorphisms. Finally, let us observe that if a triple $(G, \varphi, F)$ as in Definition 1 is 2-exact, then

$$
\pi_{0}(\mathbb{K}) \stackrel{\pi_{0}(G)}{\longrightarrow} \pi_{0}(\mathbb{G}) \stackrel{\pi_{0}(F)}{\longrightarrow} \pi_{0}(\mathbb{H}) \quad \pi_{1}(\mathbb{K}) \stackrel{\pi_{1}(G)}{\longrightarrow} \pi_{1}(\mathbb{G}) \stackrel{\pi_{1}(F)}{\longrightarrow} \pi_{1}(\mathbb{H})
$$

are exact sequences of pointed sets and groups.

Consider now a pointed topological space $Y$ and its fundamental groupoid $\Pi_{1}(Y)$, i.e. the pointed groupoid having points of $Y$ as objects and homotopy rel end-points classes of paths as arrows (we use the additive notation for the concatenation of paths). This construction gives rise to a functor

$$
\Pi_{1}: \operatorname{Top}_{*} \rightarrow G p d_{*}
$$

between the category of pointed topological spaces and the category of pointed groupoids. Recall, from [3], the following lemma.

Lemma 2. The functor $\Pi_{1}$ is homotopy equivariant. In particular, if $f: X \rightarrow Y$ is a homotopy equivalence in $T o p_{*}$, then $\Pi_{1}(f)$ is an equivalence in $G p d_{*}$.

In fact, $\Pi_{1}: T o p_{*} \rightarrow G p d_{*}$ is a 2-functor, when we take as 2-cells in $T o p_{*}$ homotopy classes of homotopies. Moreover, for any $f$ in $T o p_{*}, \Pi_{1}(f)$ is a strict morphism in $G p d_{*}$. 


\section{The exact sequence}

Consider a map in $T o p_{*}$ together with its homotopy kernel

$$
K f \stackrel{k f}{\longrightarrow} X \stackrel{f}{\longrightarrow} Y
$$

Recall that $K f$ is the subspace of the product space $X \times Y^{I}$ given by the pairs $(x, \eta: * \rightarrow f(x))$, with $x$ a point of $X, \eta$ a path in $Y$ and $*$ the base point.

Proposition 3. The sequence

$$
\Pi_{1}(K f) \stackrel{\Pi_{1}(k f)}{\longrightarrow} \Pi_{1}(X) \stackrel{\Pi_{1}(f)}{\longrightarrow} \Pi_{1}(Y),
$$

with the pointed natural transformation

$$
\varphi: 0 \Rightarrow \Pi_{1}(k f) \cdot \Pi_{1}(f) \quad \varphi_{(x, \eta)}=[\eta]: * \rightarrow f(x),
$$

is 2-exact.

Proof. Consider the following commutative diagram in $G p d_{*}$, where $f^{\prime}$ is the comparison morphism

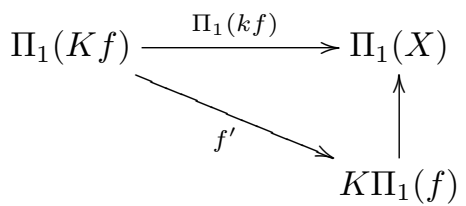

Following the general description given in the first section, we obtain the following explicit description for $\Pi_{1}(K f), K \Pi_{1}(f)$ and $f^{\prime}$ :

- an object of $\Pi_{1}(K f)$ is a pair $(x, \eta: * \rightarrow f(x))$ in $X \times Y^{I}$;

- an arrow $[h, H]:(x, \eta) \rightarrow\left(x^{\prime}, \eta^{\prime}\right)$ in $\Pi_{1}(K f)$ is a class of pairs with $h: x \rightarrow x^{\prime}$ a path in $X$ and $H: I \rightarrow Y^{I}$ such that $H(0)=\eta, H(1)=\eta^{\prime}$ and, for all $t$ in $I, H(t): * \rightarrow f(h(t))$ a path in $Y$;

- an object of $K \Pi_{1}(f)$ is a pair $(x,[\eta]: * \rightarrow f(x))$, where $[\eta]$ is a map in $\Pi_{1}(Y)$, i.e. a class of paths $\eta: * \rightarrow f(x)$ in $Y$;

- an arrow $[h]:(x,[\eta]) \rightarrow\left(x^{\prime},\left[\eta^{\prime}\right]\right)$ in $K \Pi_{1}(f)$ is a class of paths $h: x \rightarrow x^{\prime}$ in $X$ such that the following diagram in $\Pi_{1}(Y)$ commutes

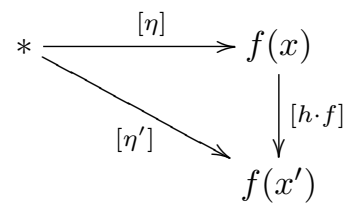

- the functor $f^{\prime}$ sends $[h, H]:(x, \eta) \rightarrow\left(x^{\prime}, \eta^{\prime}\right)$ to $[h]:(x,[\eta]) \rightarrow\left(x^{\prime},\left[\eta^{\prime}\right]\right)$.

Clearly, $f^{\prime}$ is (essentially) surjective on objects. Moreover, consider an arrow $[h]: f^{\prime}(x, \eta) \rightarrow f^{\prime}\left(x^{\prime}, \eta^{\prime}\right)$ in $K \Pi_{1}(f)$. The commutativity of the previous diagram gives us a continuous map $L: I \times I \rightarrow Y$ such that $L(0, t)=(\eta+h \cdot f)(t), L(1, t)=$ 
$\eta^{\prime}(t), L(s, 0)=*, L(s, 1)=f(h(s))$ for all $s, t$ in $I$. By a well-known transformation (studied abstractly in [8], under the name of "lens conversion") one can derive from $L$ a map $N: I \times I \rightarrow Y$ such that $N(0, t)=\eta(t), N(1, t)=\eta^{\prime}(t), N(s, 0)=$ $*, N(s, 1)=f(h(s))$ for all $s, t$ in $I$. Finally, put $H: I \rightarrow Y^{I} H(s)=N(s,-)$. In this way, we have an arrow $[h, H]:(x, \eta) \rightarrow\left(x^{\prime}, \eta^{\prime}\right)$ in $\Pi_{1}(K f)$ such that $f^{\prime}([h, H])=[h]$, that is $f^{\prime}$ is full.

In the next corollary, we write $\Omega: T o p_{*} \rightarrow T o p_{*}$ for the loop-space endofunctor and $\Pi_{n+1}(Y)$ for the pointed groupoid $\Pi_{1}\left(\Omega^{n} Y\right)$.

Corollary 4. Let $f: X \rightarrow Y$ be a map in Top ${ }_{*}$ : there is a long 2-exact sequence of pointed groupoids

$$
\begin{aligned}
\ldots & \rightarrow \Pi_{n}(K f) \rightarrow \Pi_{n}(X) \rightarrow \Pi_{n}(Y) \rightarrow \ldots \\
\ldots \rightarrow \Pi_{2}(K f) & \rightarrow \Pi_{2}(X) \rightarrow \Pi_{2}(Y) \rightarrow \Pi_{1}(K f) \rightarrow \Pi_{1}(X) \rightarrow \Pi_{1}(Y)
\end{aligned}
$$

Proof. It is enough to recall that the dual Puppe sequence [15]

$$
\begin{gathered}
\ldots \rightarrow \Omega^{n}(K f) \rightarrow \Omega^{n}(X) \rightarrow \Omega^{n}(Y) \rightarrow \ldots \\
\ldots \rightarrow \Omega(K f) \rightarrow \Omega(X) \rightarrow \Omega(Y) \rightarrow K f \rightarrow X \rightarrow Y
\end{gathered}
$$

is homotopy equivalent to the sequence of iterated homotopy kernels

$$
\cdots \longrightarrow K(k(k f)) \stackrel{k(k(k f))}{\longrightarrow} K(k f) \stackrel{k(k f)}{\longrightarrow} K f \stackrel{k f}{\longrightarrow} X \stackrel{f}{\longrightarrow} Y
$$

(as proved in a general, abstract setting in [7]). Since to be full and essentially surjective is stable under composition with equivalences, we can apply Proposition 3 to each point of the kernel sequence and, by Lemma 2, we obtain the required long 2-exact sequence.

\section{Remarks :}

1) Clearly, $\pi_{0}\left(\Pi_{1}(Y)\right)=\pi_{0}(Y)$ and $\pi_{1}\left(\Pi_{1}(Y)\right)=\pi_{1}(Y)$; more generally, $\pi_{0}\left(\Pi_{n+1}(Y)\right)=\pi_{n}(Y)$ and $\pi_{1}\left(\Pi_{n+1}(Y)\right)=\pi_{n+1}(Y)$. As a consequence, applying the functor $\pi_{0}: G p d_{*} \rightarrow S e t_{*}$ to the 2-exact sequence of Corollary 4, we obtain the usual homotopy exact sequence

$$
\ldots \rightarrow \pi_{1}(K f) \rightarrow \pi_{1}(X) \rightarrow \pi_{1}(Y) \rightarrow \pi_{0}(K f) \rightarrow \pi_{0}(X) \rightarrow \pi_{0}(Y) .
$$

Applying the functor $\pi_{1}: G p d_{*} \rightarrow$ Groups, we obtain the same sequence, but we miss the three terms of degree zero.

2) In [6], A. Garzon, J. Miranda and A. del Río show that the groupoid $\Pi_{n}(Y)$ is a cat-group for $n \geqslant 2$, a braided cat-group for $n \geqslant 3$ and a symmetric cat-group for $n \geqslant 4$. Moreover, if $f: X \rightarrow Y$ is in $T o p_{*}$ and $n \geqslant 2, \Pi_{n}(f)$ is a monoidal functor (compatible with the braiding if $n \geqslant 3$ ). Since the definition of 2-exactness remains unchanged passing from pointed groupoids to (eventually braided or symmetric) cat-groups, the 2-exact sequence of Corollary 4 is in fact a 2-exact sequence of cat-groups for $n \geqslant 2$. 


\section{Strong exactness}

Let $A$ and $B$ be two groups. To give a homomorphism $f: A \rightarrow B$ is equivalent to giving an action of the group $A$ on the underlying set $B$ which also satisfies a supplementary condition (iii), namely a mapping $+: A \times B \rightarrow B$ such that

(i) $0_{A}+b=b$

(ii) $\left(a_{1}+a_{2}\right)+b=a_{1}+\left(a_{2}+b\right)$

(iii) $a+\left(b_{1}+b_{2}\right)=\left(a+b_{1}\right)+b_{2}$

for all $a, a_{1}, a_{2} \in A$ and for all $b, b_{1}, b_{2} \in B$. Indeed, given the action $+: A \times B \rightarrow B$, we get $f: A \rightarrow B$ by $f(a)=a+0_{B}$. Conversely, given $f: A \rightarrow B$, we put $a+b=$ $f(a)+b$. Moreover, given a morphism $g: B \rightarrow C$ in Groups, the following conditions are equivalent:

(1) the composite $f \cdot g$ is equal to the zero morphism;

(2) the following diagram, where $p_{B}$ is the projection, commutes

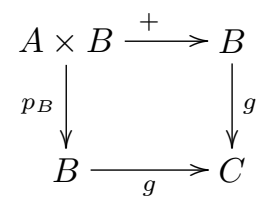

Finally, the following conditions are equivalent:

(I) if $g(b)=0_{C}$, then there is $a \in A$ such that $b=f(a)$;

(II) if $g\left(b_{1}\right)=g\left(b_{2}\right)$, then there is $a \in A$ such that $b_{1}=a+b_{2}$.

Assume now that $A$ is a group and $B$ and $C$ are just pointed sets. Still, given a map $+: A \times B \rightarrow B$ such that (i) and (ii) hold, we obtain a morphism $f: A \rightarrow B$ in $\operatorname{Set}_{*}$ by $f(a)=a+0_{B}$ (the opposite construction does not make sense). But now condition (2) is stronger than condition (1) and condition (II) is stronger than condition (I). The strong conditions can be expressed by means of the pointed orbit set $B / A$, a sort of "cokernel" of the action $+: A \times B \rightarrow B$. In fact, the projection $P_{+}: B \rightarrow B / A$ is the coequalizer of + and $p_{B}$ (within pointed sets). Now, a mapping $g: B \rightarrow C$ satisfies (2) iff it factors through $P_{+}$; it also satisfies (II) iff the comparison mapping $B / A \rightarrow C$ is surjective.

The interest of the strong exactness condition (II) comes from the homotopy sequence: if $f: X \rightarrow Y$ is in $T o p_{*}$, there is a well-known action of the $H$-space $\Omega Y$ on $K f$

$$
+: \Omega Y \times K f \rightarrow K f ; \quad \omega+(x, \eta)=(x, \omega+\eta)
$$

which induces a map $+: \pi_{0}(\Omega Y) \times \pi_{0}(K f) \rightarrow \pi_{0}(K f)$ such that conditions (i), (ii), (2) and (II) hold (see [2] for a detailed discussion).

The rest of this section is devoted to study the 2-dimensional analogue of strong exactness. Let $\mathbb{A}$ be a cat-group and $\mathbb{B}$ a pointed groupoid and consider a functor $\mu: \mathbb{A} \times \mathbb{B} \rightarrow \mathbb{B}$ together with two natural isomorphisms $m_{B}^{0}: B \rightarrow \mu(O, B)$ and $m_{A_{1}, A_{2}, B}: \mu\left(A_{1} \otimes A_{2}, B\right) \rightarrow \mu\left(A_{1}, \mu\left(A_{2}, B\right)\right)$, coherent with respect to the catgroup structure of $\mathbb{A}$. (This is equivalent to giving a monoidal functor from $\mathbb{A}$ to 
the monoidal category of endofunctors of $\mathbb{B}$.) Starting from $\left(\mu, m^{0}, m\right): \mathbb{A} \times \mathbb{B} \rightarrow \mathbb{B}$, we can construct a new pointed groupoid $\operatorname{Cok} \mu$ in the following way:

- the objects of $C o k \mu$ are those of $\mathbb{B}$;

- a pre-morphism $(A, f): B_{1} \rightarrow B_{2}$ in $\operatorname{Cok} \mu$ is a pair with $A$ in $\mathbb{A}$ and $f: B_{1} \rightarrow$ $\mu\left(A, B_{2}\right)$ in $\mathbb{B}$;

- a morphism $[A, f]: B_{1} \rightarrow B_{2}$ is an equivalence class of pre-morphisms: two pre-morphisms $(A, f),\left(A^{\prime}, f^{\prime}\right): B_{1} \rightarrow B_{2}$ are equivalent if there is $\alpha: A \rightarrow A^{\prime}$ such that $f \cdot \mu\left(\alpha, B_{2}\right)=f^{\prime}$;

- the base point of $\operatorname{Cok} \mu$ is that of $\mathbb{B}$.

There is a morphism of pointed groupoids $P_{\mu}: \mathbb{B} \rightarrow \operatorname{Cok} \mu$ which sends $g: B_{1} \rightarrow B_{2}$ to $\left[0, g \cdot m_{B_{2}}^{0}\right]: B_{1} \rightarrow B_{2}$. There is also a natural transformation

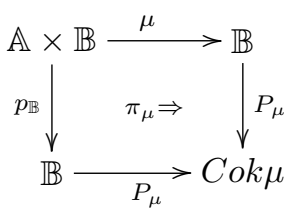

given by $\pi_{\mu}(A, B)=\left[A^{*}, m_{B}^{0} \cdot m_{A^{*}, A, B}: B \rightarrow \mu(0, B) \simeq \mu\left(A^{*} \otimes A, B\right) \rightarrow\right.$ $\left.\mu\left(A^{*}, \mu(A, B)\right)\right]: B \rightarrow \mu(A, B)$ (where $A^{*}$ is a dual of $A$ ). Moreover, the following diagrams in $C o k \mu$ commute

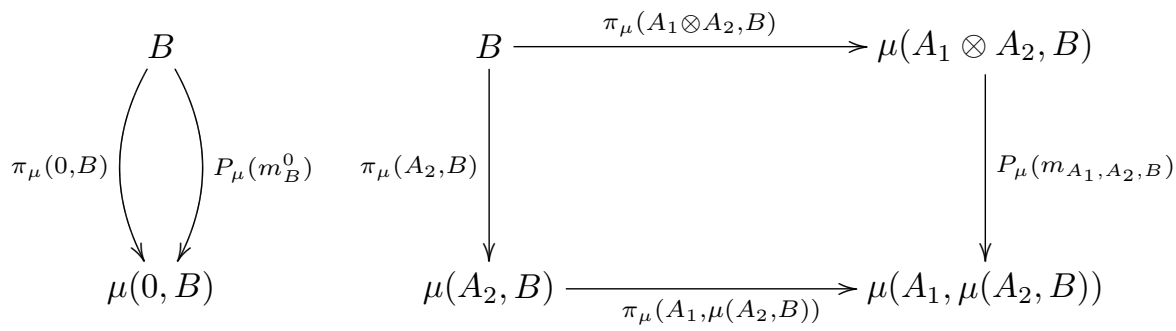

The previous construction is universal in the following sense (Cok $\mu$ is the isocoinserter of $\mu$ and $\left.p_{\mathbb{B}}\right)$ : given

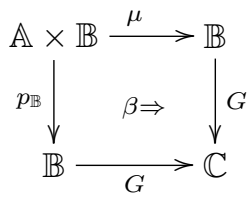


with $G: \mathbb{B} \rightarrow \mathbb{C}$ in $G p d_{*}$ and $\beta$ a natural transformation such that

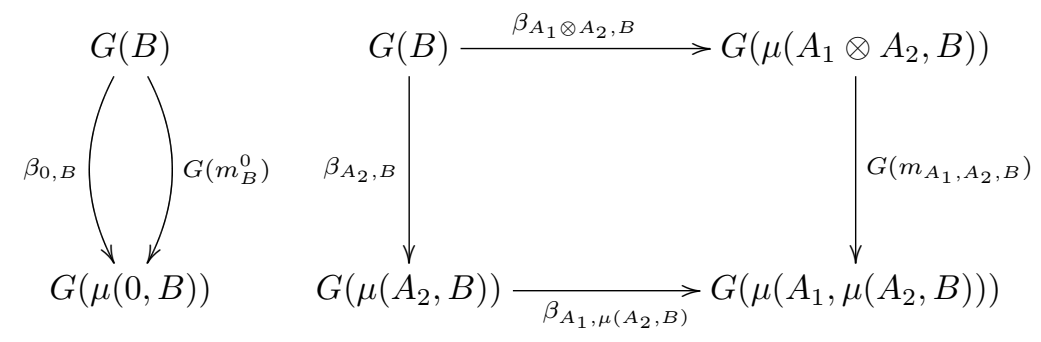

commute, there is a unique $G^{\prime}: \operatorname{Cok} \mu \rightarrow \mathbb{C}$ in $G p d_{*}$ such that $P_{\mu} \cdot G^{\prime}=G$ and $\pi_{\mu} \cdot G^{\prime}=\beta$.

Proof. One has to define $G^{\prime}: \operatorname{Cok} \mu \rightarrow \mathbb{C}$ by

$$
G^{\prime}:[A, f]: B_{1} \rightarrow B_{2} \mapsto G(f) \cdot \beta_{A, B_{2}}^{-1}: G\left(B_{1}\right) \rightarrow G\left(\mu\left(A, B_{2}\right)\right) \rightarrow G\left(B_{2}\right)
$$

The uniqueness follows from the commutativity of the following diagram

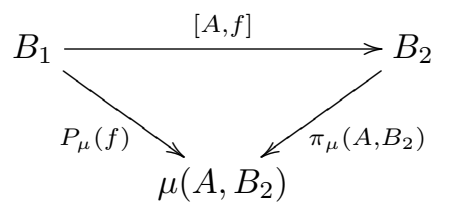

This universal property characterizes Cok u up to isomorphism. The triple $\left(\operatorname{Cok} \mu, P_{\mu}, \pi_{\mu}\right)$ has also a "biuniversal" property (it is the bi-coequaliser of $\mu$ and $\left.p_{\mathbb{B}}\right)$ which characterizes it up to equivalence. It is similar to those of the kernel and of the cokernel (see $[\mathbf{1 1}, \mathbf{1 6}])$.

Let us explain the notation $\operatorname{Cok} \mu$. Starting from $\left(\mu, m^{0}, m\right): \mathbb{A} \times \mathbb{B} \rightarrow \mathbb{B}$, we get a morphism $F: \mathbb{A} \rightarrow \mathbb{B}$ in $G p d_{*}$ by $F(A)=\mu(A, 0)$ and $f_{0}=m_{0}^{0}: 0 \rightarrow \mu(0,0)=F(0)$. If it is the case that $\mathbb{A}$ and $\mathbb{B}$ are symmetric cat-groups and $F$ is a monoidal functor compatible with the symmetry, then $C o k \mu$ is exactly the cokernel of $F$ as described in $[11,16]$.

In order to state strong exactness for pointed groupoids, observe that, given

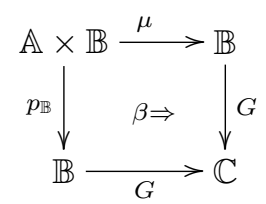

as before, we get a pointed natural transformation

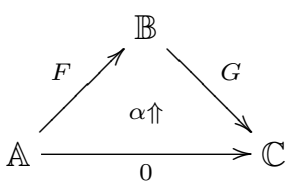


by $\alpha_{A}=g_{0} \cdot \beta_{A, 0}: 0 \rightarrow G(0) \rightarrow G(\mu(A, 0))=G(F(A))$. Recall now that, if $(F, \alpha, G)$ is a sequence of symmetric cat-groups, its 2-exactness can be equivalently stated by asking that the canonical comparison from the cokernel of $F$ to $G$ is full and faithful (Proposition 6.2 in [11]). With this fact in mind, we give the following definition.

Definition 5. Consider

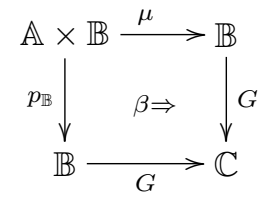

as before. The sequence $(\mu, \beta, G)$ is strongly 2-exact if the comparison functor $G^{\prime}: \operatorname{Cok} \mu \rightarrow \mathbb{C}$ is full and faithful.

Here is the expected link between strong 2-exactness (Definition 5) and 2-exactness (Definition 1). The proof is a direct calculation.

Proposition 6. Consider

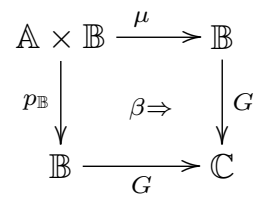

and

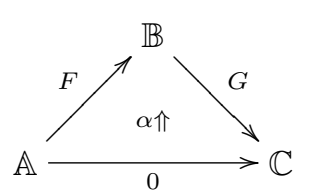

as before. Consider also the factorization $F^{\prime}$ of $F$ through the kernel of $G$ and the factorization $G^{\prime}$ of $G$ through $C o k \mu$

$$
F^{\prime}: \mathbb{A} \rightarrow K G \quad G^{\prime}: \operatorname{Cok} \mu \rightarrow \mathbb{C} .
$$

a) If $G^{\prime}$ is faithful, then $F^{\prime}$ is full;

b) If $G^{\prime}$ is full, then $F^{\prime}$ is essentially surjective.

The interested reader can verify that, if $\mathbb{A}, \mathbb{B}, \mathbb{C}, F, G$ and $\alpha$ belong to the 2-category of cat-groups and if $\beta$ is compatible with the cat-group structure of $\mathbb{B}$ and $\mathbb{C}$, then both the implications of Proposition 6 can be reversed.

Let us write down explicitly the condition of fullness for $G^{\prime}:$ Cok $\mu \rightarrow \mathbb{C}:$ given two objects $B_{1}$ and $B_{2}$ in $\mathbb{B}$ and an arrow $c: G\left(B_{1}\right) \rightarrow G\left(B_{2}\right)$ in $\mathbb{C}$, there is $A$ in $\mathbb{A}$ and $f: B_{1} \rightarrow \mu\left(A, B_{2}\right)$ in $\mathbb{B}$ such that $G^{\prime}[A, f]=c$.

The analogy between fullness of $G^{\prime}$ and strong exactness for a sequence of groups and pointed sets, i.e. condition (II), is now clear. This analogy is made more precise in the next remark.

Remark :

1) Assume that the categories $\mathbb{A}, \mathbb{B}$ and $\mathbb{C}$ of Proposition 6 are discrete (so that $\mathbb{A}$ is a group, and $\mathbb{B}$ and $\mathbb{C}$ are pointed sets, but $\operatorname{Cok} \mu$ is not discrete). Then the fullness of $G^{\prime}$ is exactly condition (II) (moreover, the faithfulness of $G^{\prime}$ implies that $F$ is injective).

2) Assume that the categories $\mathbb{A}, \mathbb{B}$ and $\mathbb{C}$ of Proposition 6 have a unique object (so that $\mathbb{A}$ is an abelian group and $\mathbb{B}$ and $\mathbb{C}$ are groups). Then the faithfulness of $G^{\prime}$ 
is exactly condition (II) (moreover, the fullness of $G^{\prime}$ is equivalent to the surjectivity of $G)$.

Finally, we come back to the higher dimensional homotopy sequence. Let $f: X \rightarrow$ $Y$ be an arrow in $T o p_{*}$ and consider the action $\Omega Y \times K f \rightarrow K f$ as at the beginning of this section. It induces an action $\mu: \Pi_{1}(\Omega Y) \times \Pi_{1}(K f) \rightarrow \Pi_{1}(K f)$ of the catgroup $\Pi_{2}(Y)=\Pi_{1}(\Omega Y)$ on the pointed groupoid $\Pi_{1}(K f)$. Moreover, the diagram

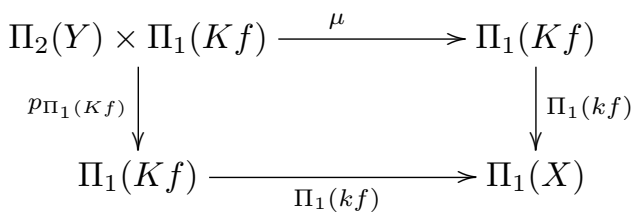

is strictly commutative.

Proposition 7. Let

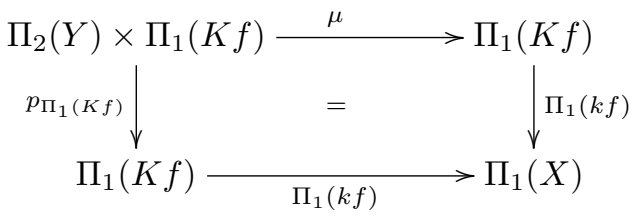

be as before. The sequence $\left(\mu,=, \Pi_{1}(k f)\right)$ is strongly 2-exact.

We leave the proof as an exercise for the reader.

\section{References}

[1] F. Borceux: Handbook of categorical algebra, Cambridge University Press (1994).

[2] R. Brown: Fibrations of groupoids, Journal of Algebra 15 (1970) 103132 .

[3] R. Brown: Topology: A geometric account of general topology, homotopy types and the fundamental groupoid, Ellis Horwood Ltd. (1988).

[4] R. BRown: Groupoids and crossed objects in algebraic topology, Homology, Homotopy and Applications 1 (1999) 1-78 (available at http://www.rmi.acnet.ge/hha/).

[5] P. Carrasco, A.M. Cegarra: (Braided) tensor structures on homotopy groupoids and nerves of (braided) categorical groups, Communications in Algebra 24 (1996) 3995-4058.

[6] A.R. Garzon, J.G. Miranda, A. DEL Río: Tensor structures on homotopy groupoids of topological spaces, Intern. Math. Journal 2 (2002) 407-431.

[7] M. GRAndis: Homotopical algebra in homotopical categories, Appl. Categ. Struct. 2 (1994) 351-406. 
[8] M. Grandis: Categorically algebraic foundations for homotopical algebra, Appl. Categ. Struct. 5 (1997) 363-413.

[9] M. Grandis: A note on exactness and stability in homotopical algebra, Theory and Applications of Categories 9 (2001) 17-42 (available at http://www.tac.mta.ca/tac/).

[10] A. Joyal, R. Street: Braided tensor categories, Adv. Math. 102 (1993) 20-78.

[11] S. Kasangian, E.M. Vitale: Factorization systems for symmetric cat-groups, Theory and Applications of Categories 7 (2000) 47-70 (available at http://www.tac.mta.ca/tac/).

[12] R.W. Kieboom, E.M. Vitale: On the exact sequence associated to a fibration of 2-groupoids, preprint (2000).

[13] M. Mather: Pull-backs in homotopy theory, Can. J. Math. 28 (1976) 225-263.

[14] R.A. Piccinini: Lectures on homotopy theory, North-Holland (1992).

[15] D. Puppe: Homotopiemengen und ihre induzierten Abbildungen, I, Math. Z. 69 (1958) 299-344, 395-417.

[16] E.M. Vitale: A Picard-Brauer exact sequence of categorical groups, J. Pure Appl. Algebra (to appear).

[17] G.W. Whitenead: Elements of homotopy theory, Springer Verlag (1978).

This article may be accessed via WWW at http://www.rmi.acnet.ge/hha/ or by anonymous ftp at

$$
\text { ftp://ftp.rmi.acnet.ge/pub/hha/volumes/2002/n1a5/v4n1a5.(dvi,ps,pdf) }
$$

M. Grandis grandis@dima.unige.it

Dipartimento di Matematica, Università di Genova,

Via Dodecaneso 35, 16146 Genova, Italy

E. M. Vitale vitale@math.ucl.ac.be

Département de Mathématique, Université catholique de Louvain, Chemin du Cyclotron 2, 1348 Louvain-la-Neuve, Belgium 